\title{
Enrichment of inflammatory bowel disease and colorectal cancer risk variants in colon expression quantitative trait loci
}

\author{
Imge Hulur ${ }^{1}$, Eric R Gamazon ${ }^{2,6}$, Andrew D Skol ${ }^{2}$, Rosa M Xicola ${ }^{4}$, Xavier Llor ${ }^{4}$, Kenan Onel ${ }^{3}$, Nathan A Ellis ${ }^{5}$ \\ and Sonia S Kupfer ${ }^{2 *}$
}

\begin{abstract}
Background: Genome-wide association studies (GWAS) have identified single nucleotide polymorphisms (SNPs) associated with diseases of the colon including inflammatory bowel diseases (IBD) and colorectal cancer (CRC). However, the functional role of many of these SNPs is largely unknown and tissue-specific resources are lacking. Expression quantitative trait loci (eQTL) mapping identifies target genes of disease-associated SNPs. This study provides a comprehensive eQTL map of distal colonic samples obtained from 40 healthy African Americans and demonstrates their relevance for GWAS of colonic diseases.
\end{abstract}

Results: 8.4 million imputed SNPs were tested for their associations with 16,252 expression probes representing 12,363 unique genes. 1,941 significant cis-eQTL, corresponding to 122 independent signals, were identified at a false discovery rate (FDR) of 0.01 . Overall, among colon cis-eQTL, there was significant enrichment for GWAS variants for IBD (Crohn's disease [CD] and ulcerative colitis [UC]) and CRC as well as type 2 diabetes and body mass index. ERAP2, ADCY3, INPP5E, UBA7, SFMBT1, NXPE1 and REXO2 were identified as target genes for IBD-associated variants. The CRC-associated eQTL rs3802842 was associated with the expression of C11orf93 (COLCA2). Enrichment of colon eQTL near transcription start sites and for active histone marks was demonstrated, and eQTL with high population differentiation were identified.

Conclusions: Through the comprehensive study of eQTL in the human colon, this study identified novel target genes for IBD- and CRC-associated genetic variants. Moreover, bioinformatic characterization of colon eQTL provides a tissue-specific tool to improve understanding of biological differences in diseases between different ethnic groups.

Keywords: Expression quantitative trait loci, Colon, Gene expression, African Americans, Regulatory variation, Transcriptomics, Inflammatory bowel disease, Colorectal cancer, Genomics, Genome-wide association studies

\section{Background}

Genetic susceptibility is thought to play a role in common diseases including those affecting the colon such as inflammatory bowel diseases (IBD) and colorectal cancer (CRC). Indeed, genome-wide association studies (GWAS), conducted primarily in populations of European descent, have identified single nucleotide polymorphisms (SNPs) associated with IBD, including both ulcerative colitis (UC) [1-6] and Crohn's disease (CD) [7-19], as well as CRC

\footnotetext{
*Correspondence: skupfer@medicine.bsd.uchicago.edu

2Department of Medicine, 900 East 57th Street, MB\#9, Chicago, IL 60637, USA Full list of author information is available at the end of the article
}

[20-30]. As is the case for GWAS variants in general, a number of these variants are located in gene deserts and their functional roles in disease pathogenesis are largely unknown [31]. Unraveling the functional basis of complex diseases is a priority as this has implications for understanding disease pathogenesis as well as identifying novel therapeutic targets [32].

Studying the genetics of gene expression is a tool that can help elucidate the functional consequences of GWAS variants. Expression quantitative trait loci (eQTL) mapping associates genome-wide SNPs with mRNA expression from the same individuals in a particular tissue to 
identify regulatory variation [33]. Previous studies [34-38] have detected eQTL in modest sample sizes (compared to typical disease GWAS). These studies have provided important insights into the architecture of gene regulation in general [39], as well as across populations [40,41] and tissues [41-43]. Importantly, it has been shown that variants identified by GWAS as reproducibly associated with complex traits are enriched for eQTL in various cell types $[33,44,45]$.

While eQTL mapping studies have been performed in lymphoblastoid cell lines (LCLs) [36,37,40,42,46], liver $[35,47]$, adipose tissue [42], brain [48], skin $[42,46]$ and ileum [49], these tissue types may not be relevant for all disease traits. A recent eQTL mapping study in the human ileum noted tissue-specific effects as well as enrichment for IBD susceptibility variants [49]. More recently, Closa et al. conducted an eQTL analysis of CRC loci in colonic mucosa and found significant cis-eQTL in three loci [50]. However, comprehensive genome-wide eQTL mapping has not previously been performed in the human colon, which is the relevant tissue for colonic diseases like IBD and CRC.

The goal of the present study was to comprehensively map eQTL in healthy human colon in order to characterize colon-specific gene regulation and evaluate its relevance for GWAS of IBD and CRC as well as other complex phenotypes. Towards this end, genome-wide genotyping, using a microarray optimized for individuals of African descent, and gene expression profiling were performed colonic tissue obtained from 40 African American (AA) healthy subjects who underwent screening colonoscopies. These findings and resources will allow for improved understanding of disease pathogenesis of inflammatory and malignant diseases of the colon.

\section{Results}

In order to identify significant colon eQTL, the genotype and expression data was first subjected to a number of quality controls. Using the cleaned data, cis- and transeQTL were identified. The overlap between colon eQTL and disease-associated GWAS SNPs was performed as well as the overlap with eQTL in other tissue types. As only AA were used in this study, population differentiated eQTL were also identified. Finally, using simulations, enrichment of colon eQTL among IBD- and CRC-associated variants and for histone marks was performed. Results for these analyses are presented in this order in the sections that follow.

\section{Quality control of genotype and expression data}

2.2 million SNPs were genotyped in 48 AA subjects using the Affymetrix Axiom Pan-African array (Figure 1A). SNPs were removed if they had a genotyping rate $<95 \%(\mathrm{n}=$ $62,060)$, minor allele frequency $(\mathrm{MAF})<0.05(\mathrm{n}=611,784)$, significant Hardy-Weinberg equilibrium (HWE) p-values $(\mathrm{n}=3,395$, see Methods), non-autosomal SNPs $(\mathrm{n}=46,486)$, or mapped to the same position $(\mathrm{n}=722)$ as another SNP. Imputation was performed using 1.492 million remaining SNPs and the 1000 Genomes Project reference panel Phase I integrated variant set release (v3), which provided a final dataset of 8.4 million SNPs, after removing imputed SNPs with $\mathrm{MAF}<0.05$ or low imputation quality (IMPUTE2-info score $<0.5$ ) (Figure 1B). Principal components analysis (PCA) showed that subjects included in this study were similar to individuals of African ancestry in Southwest USA (ASW) HapMap population samples in terms of ancestry (Additional file 1). The proportion of European and African ancestry among the subjects were represented by the first principal component (PC); PC1 was thus used as a covariate in the eQTL analyses to control for global ancestry.

In gene expression analysis, 47,231 probes were profiled. A total of 16,252 probes remained for analysis after removing probes that mapped to more than one genomic location $(n=19,278)$, that contained one or more SNP $(n=2,343)$, or that was not expressed in one or more subjects $(n=9,358)$. Heat map visualization and hierarchical clustering of the gene expression data suggested the removal of eight outliers, leaving 40 individuals (Additional file 2). Inclusion of the first five $\mathrm{PCs}$ of the gene expression data yielded the maximal number of significant cis-eQTL probes at various false discovery rate (FDR) thresholds (Additional file 3). These expression-based PCs were included as covariates in subsequent eQTL analysis to correct for unmeasured yet systematic variation in gene expression levels.

\section{Identification of colon eQTL}

eQTL were identified by testing for an association between each SNP and each gene's expression probe. The distribution of eQTL p-values was compared against the distribution expected by chance separately for cis- and trans-eQTL. For cis-, but not trans-eQTL, we found a significant enrichment for small p-values (Additional file 4). We identified 1,941 cis-eQTL corresponding to 122 genes at a FDR of 0.01 , which represent 122 independent SNP-gene associations (Table 1). Cis-eQTL were found to be highly enriched around transcription start sites (TSS) with no discernible trend toward $3^{\prime}$ or 5' (Additional file 5).

\section{Overlap of cis-eQTL with colonic disease-associated GWAS variants}

The National Human Genome Research Institute (NHGRI) GWAS catalog was searched to identify IBD (i.e. CD and/ or UC)- and CRC-associated GWAS variants that are also significant cis-eQTL at FDR $<0.10$ in the colon samples. Of 8,122 significant cis-eQTL at this threshold, 4 were previously identified in GWAS of IBD (i.e. both CD and 


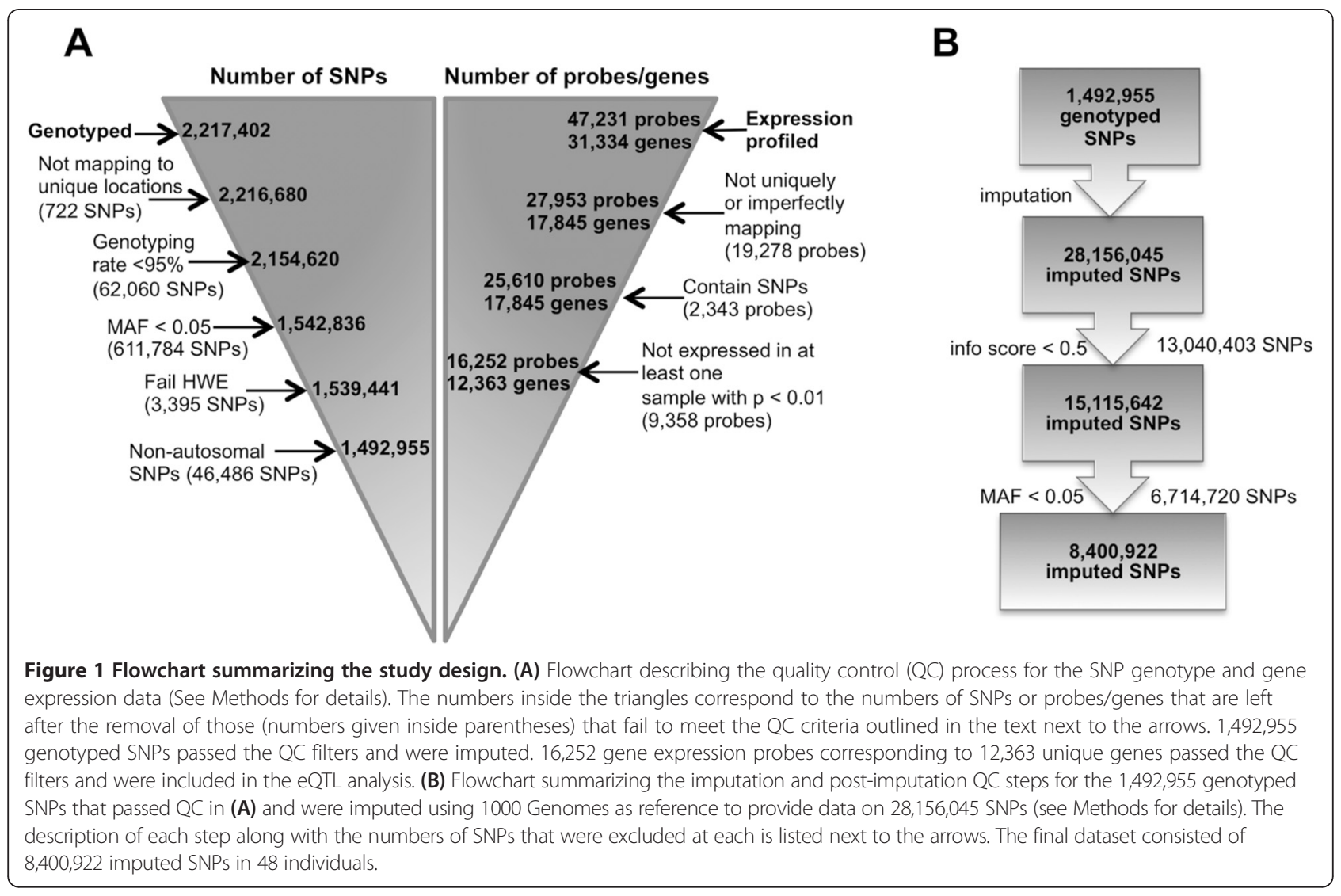

UC), CD only, UC only or CRC. When SNPs in high linkage disequilibrium (LD) $\left(\mathrm{r}^{2} \geq 0.8\right)$ with the GWAS variants were included, the overlap between GWAS signals and significant cis-eQTL increased to 127 variants: 103 common to CD and UC, 6 for UC-only and 18 for CRC. These significant cis-eQTL corresponded to 8 unique target genes that co-localize with disease-associated SNPs (Figure 2; Table 2).

\section{Comparison of colon cis-eQTL with other tissues}

A comparative analysis was performed to assess the extent of overlap between colon cis-eQTL and cis-eQTL for other tissue types (Table 3). Substantial percentages of colon cis-eQTL target genes were found to be cis-eQTL

Table 1 Numbers of significant colon cis-eQTL identified at four FDR thresholds

\begin{tabular}{lllll}
\hline & \multicolumn{4}{l}{ Number of cis-eQTL } \\
\cline { 2 - 5 } FDR & SNP-gene pairs & SNPs & Independent & Genes \\
\hline 0.01 & 1,941 & 1,941 & 122 & 122 \\
0.05 & 5,183 & 5,181 & 353 & 352 \\
0.10 & 8,252 & 8,122 & 693 & 684 \\
0.20 & 14,255 & 14,177 & 1,677 & 1,614 \\
\hline
\end{tabular}

*These numbers correspond to the numbers of independent SNP-gene associations that were determined using the stepwise association method outlined in Supplementary Methods (Additional file 8). target genes in other tissues, including genes in LCLs, liver, brain, skin, and ileum. As the FDR threshold for defining cis-eQTL in the colon became more stringent, the percentage overlap of colon cis-eQTL target genes with cis-eQTL target genes from other tissues increased.

\section{Population differentiated colon cis-eQTL}

Fixation index $\left(\mathrm{F}_{\mathrm{ST}}\right)$ values were calculated between 1000 Genomes Project European (EUR) and African (AFR) populations for all significant colon cis-eQTL (FDR $<0.20$ ). Out of the 14,177 significant cis-eQTL SNPs, $F_{\mathrm{ST}}$ estimates were successfully calculated for 14,135 SNPs. 3,185 cis-eQTL ( $23 \%$ of all cis-eQTL for which $\mathrm{F}_{\mathrm{ST}}$ values were estimated) had $\mathrm{F}_{\mathrm{ST}}>0.25$, indicating high population differentiation (Figure $3 \mathrm{~A}$ ) which was greater than that expected under the null at all FDR thresholds tested (0.2, 0.1, 0.05, 0.01; results at FDR $<0.20$ in Figure 3B). Population differentiated SNPs that are also associated with CD, $\mathrm{UC}$ and CRC were identified among colon cis-eQTL using the $\mathrm{F}_{\mathrm{ST}}$ statistic. As the mean value of $\mathrm{F}_{\mathrm{ST}}$ between CEU and YRI has been estimated to be 0.071 across 1000 Genomes Project SNPs [51], $F_{S T}$ threshold of 0.10 was used to define SNPs as population differentiated. For UC, there was one disease-associated SNP rs9847710 with $\mathrm{F}_{\mathrm{ST}}$ $>0.10$ (Additional file 6). For CD- and CRC-associated SNPs, there were no GWAS variants with $\mathrm{F}_{\mathrm{ST}}>0.10$. 


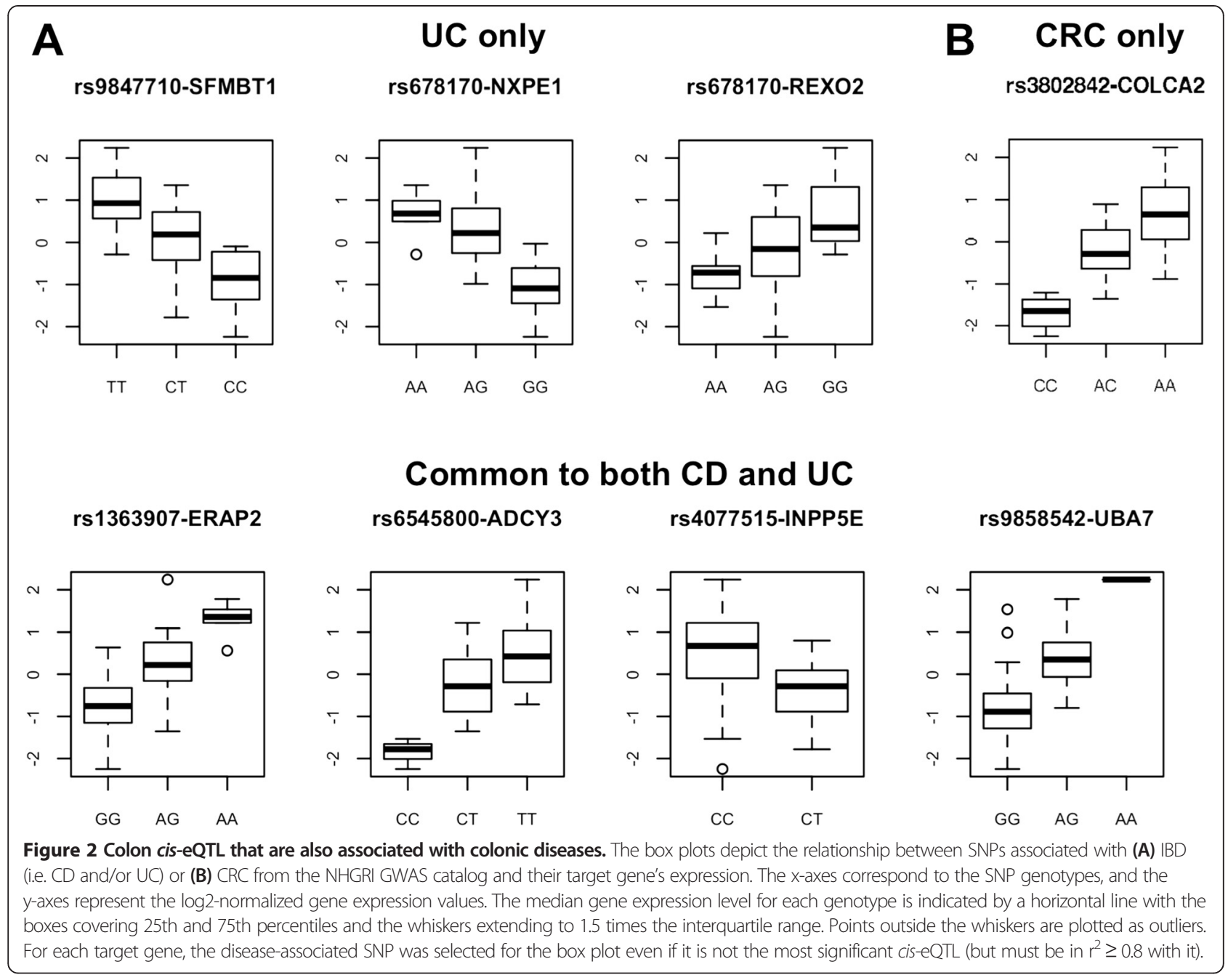

Enrichment of colon cis-eQTL among IBD- and

\section{CRC-associated variants}

Simulation-based analysis was performed to investigate whether IBD- and CRC- associated variants are enriched for the 150,213 unique cis-eQTL SNPs with $\mathrm{p}<0.001$ compared with SNPs matched on MAF (see Methods for simulation details). Significant enrichment of cis-eQTL was observed among SNPs associated with CD $(n=251)$ and UC $(n=185)$ from the GWAS catalog, with $n$ corresponding to the number of disease-associated SNPs included in the enrichment analysis, with empirical p-values for enrichment of $<0.001$ (Figure 4A; Table 4). Cis-eQTL

Table 2 Colon cis-eQTL that are associated with Crohn's disease (CD) and/or ulcerative colitis (UC) as well as colorectal cancer (CRC)

\begin{tabular}{llll}
\hline & SNP/gene & Proxy SNP* $\left(\mathbf{r}^{2}\right)$ & eQTL p-value \\
\hline Both CD and UC & rs1363907/ERAP2 & & $1.4 \times 10^{-8}$ \\
& rs6545800/ADCY3 & rs2384061 $\left(r^{2}=0.82\right)$ & $1.3 \times 10^{-7}$ \\
& rs4077515/INPP5E & rs4266763 $\left(r^{2}=0.95\right)$ & $4.7 \times 10^{-6}$ \\
& rs9858542/UBA7 & rs10640 $\left(r^{2}=0.92\right)$ & $8.7 \times 10^{-6}$ \\
CD only & rs2549794/ERAP2 & & $1.5 \times 10^{-8}$ \\
UC only & rs9847710/SFMBT1 & & $8.3 \times 10^{-6}$ \\
& rs6781710/NXPE1 \& REX02 & rs661946 $\left(r^{2}=0.94\right)$ & $8.7 \times 10^{-7}$ \\
CRC & rs3802842/COLCA2 & & $1.1 \times 10^{-6}$ \\
\hline
\end{tabular}

*SNPs in LD $\left(r^{2} \geq 0.8\right)$ with disease-associated GWAS SNPs from the NHGRI catalog. $r^{2}$ corresponds to the correlation between the proxy SNP and the GWAS SNP, calculated based on the 48 African American samples in the colon dataset. 
Table 3 Comparison of colon cis-eQTL results with published studies in different tissues

\begin{tabular}{|c|c|c|c|c|c|c|}
\hline & \multirow[b]{2}{*}{ Samples } & & \multicolumn{4}{|l|}{ FDR } \\
\hline & & & 0.01 & 0.05 & 0.10 & 0.20 \\
\hline \multirow[t]{2}{*}{ LCL [41] } & \multirow{2}{*}{$\begin{array}{l}13,643 \text { genes and } \sim 2.2 \text { million SNPs in } 270 \text { HapMap } \\
\text { subjects (CEU, CHB, JPT and YRI populations) }\end{array}$} & Genes* & $25(20 \%)$ & $49(14 \%)$ & $72(11 \%)$ & $119(7.4 \%)$ \\
\hline & & SNP-gene pairs** & 130 & 182 & 298 & 361 \\
\hline \multirow[t]{2}{*}{ Liver [35] } & \multirow{2}{*}{$\begin{array}{l}34,266 \text { genes and } 782,476 \text { SNPs in liver samples } \\
\text { acquired from } 427 \text { Caucasian subjects }\end{array}$} & Genes* & $41(34 \%)$ & 95 (27\%) & $169(25 \%)$ & $346(21 \%)$ \\
\hline & & SNP-gene pairs ${ }^{* *}$ & 8 & 15 & 20 & 33 \\
\hline \multirow[t]{2}{*}{ Brain [48] } & \multirow{2}{*}{$\begin{array}{l}22,184 \text { genes and } 1,629,853 \text { SNPs in brain tissue } \\
\text { samples from } 150 \text { Caucasian subjects }\end{array}$} & Genes* & $17(14 \%)$ & $35(9.9 \%)$ & $63(9.2 \%)$ & $137(8.5 \%)$ \\
\hline & & SNP-gene pairs** & 139 & 191 & 277 & 327 \\
\hline \multirow[t]{2}{*}{ Skin [46] } & \multirow{2}{*}{$\begin{array}{l}14,500 \text { genes and } 438,670 \text { SNPs in skin samples } \\
\text { from } 57 \text { Caucasian subjects }\end{array}$} & Genes* & $24(20 \%)$ & $39(11 \%)$ & $59(8.6 \%)$ & $92(5.7 \%)$ \\
\hline & & SNP-gene pairs** & 131 & 92 & 71 & 50 \\
\hline \multirow[t]{2}{*}{ Ileum [49] } & \multirow{2}{*}{$\begin{array}{l}19,047 \text { genes and } 581,633 \text { SNPs in ileum samples } \\
\text { from } 173 \text { mostly Caucasian subjects }\end{array}$} & Genes* & $37(30 \%)$ & $83(24 \%)$ & $118(17 \%)$ & $197(12 \%)$ \\
\hline & & SNP-gene pairs** & 176 & 130 & 94 & 58 \\
\hline
\end{tabular}

*The number of target genes from other studies overlapping colon cis-eQTL target genes are listed along with the percentages of overlap (in parentheses). To calculate the percentage of overlap, the number of overlapping target genes was divided by the total number of unique significant colon cis-eQTL target genes at the corresponding FDR threshold (refer to Table 1 for the actual numbers). ${ }^{*}$ The number of SNP-gene pairs that overlap between the colon and other tissues.

counts among the CRC-associated SNPs $(n=40)$ were also significantly higher than expected based on MAF distribution ( $\mathrm{p}=0.031)$.

In order to determine whether enrichment of colon eQTL is specific to particular complex traits or diseases, enrichment analysis was performed for SNPs associated with autoimmune disorders [celiac disease, psoriasis, rheumatoid arthritis (RA)], cancers (breast cancer, prostate cancer and melanoma), lipid traits (total cholesterol and triglyceride levels), body mass index (BMI), type 2 diabetes (T2D), and psychiatric disorders [attention deficit hyperactivity disorder (ADHD), bipolar disorder (BD) and schizophrenia]. There was statistically significant colon cis-eQTL enrichment among SNPs associated with T2D $(\mathrm{p}=0.034)$ and suggestive enrichment among SNPs associated with BMI ( $\mathrm{p}=0.055)$ (Figure 4B; Table 4). SNPs associated with
A

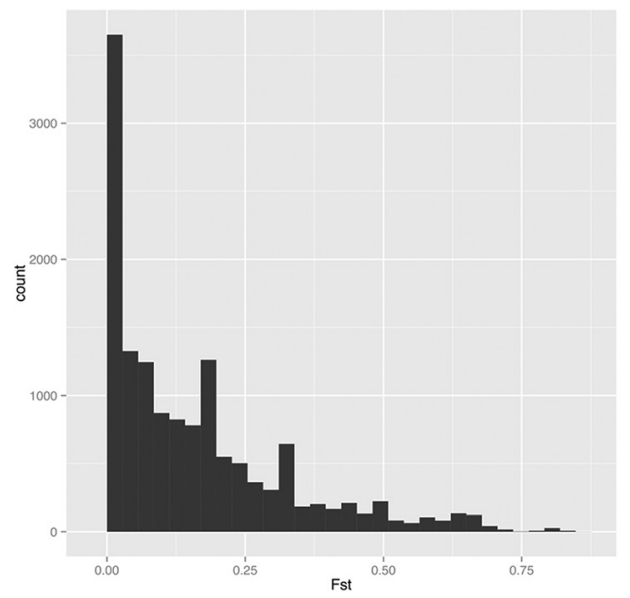

B

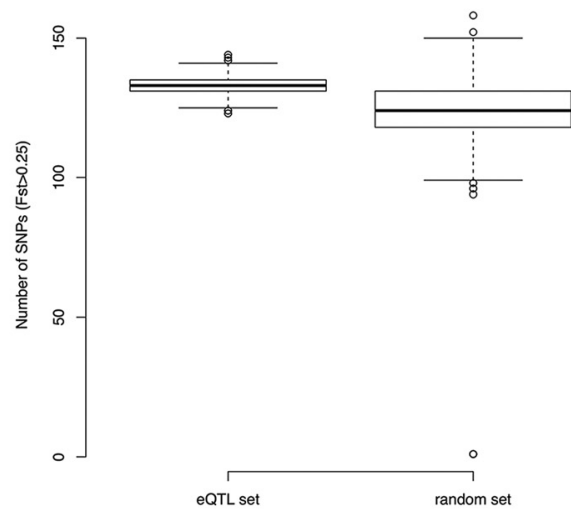

Figure 3 Cis-eQTL are enriched for SNPs that are highly differentiated between European and African populations. $F_{S T}$ values for the study SNPs were calculated between 1000 Genomes Project European (EUR) and African (AFR) populations using Weir and Cockerham's unbiased estimator. SNPS with $F_{S T}>0.25$ were defined as population differentiated SNPS. (A) The histogram shows the distribution of $F_{S T}$ values for the significant colon cis-eQTL (FDR < 0.20). Among the 14,135 cis-eQTL for which $\mathrm{F}_{\mathrm{ST}}$ estimates were obtained, 3,185 (23\%) were population differentiated. (B) Enrichment of population differentiated SNPs among significant colon cis-eQTL was evaluated using a simulation-based method. The box plot depicts the distributions of the number of population differentiated SNPs among 1,000 randomly selected cis-eQTL SNP sets (left) - generated by randomly selecting a single SNP for each unique cis-eQTL target gene $(n=684)$ among all cis-eQTL (FDR $<0.20)$ that are significantly associated with the expression of that gene-and among 1000 random sets of SNPs (right), each matching the set of 684 significant cis-eQTL SNPS, based on the distributions of MAF and distance from the nearest TSS. The numbers of population differentiated SNPs among the eQTL and random SNP sets are indicated by horizontal lines with the boxes covering 25th and 75th percentiles and the whiskers extending to 1.5 times the interquartile range. The numbers of population differentiated SNPS in the eQTL sets were significantly higher than in the random sets of SNPs ( $p<0.001$ by Mann-Whitney test). 


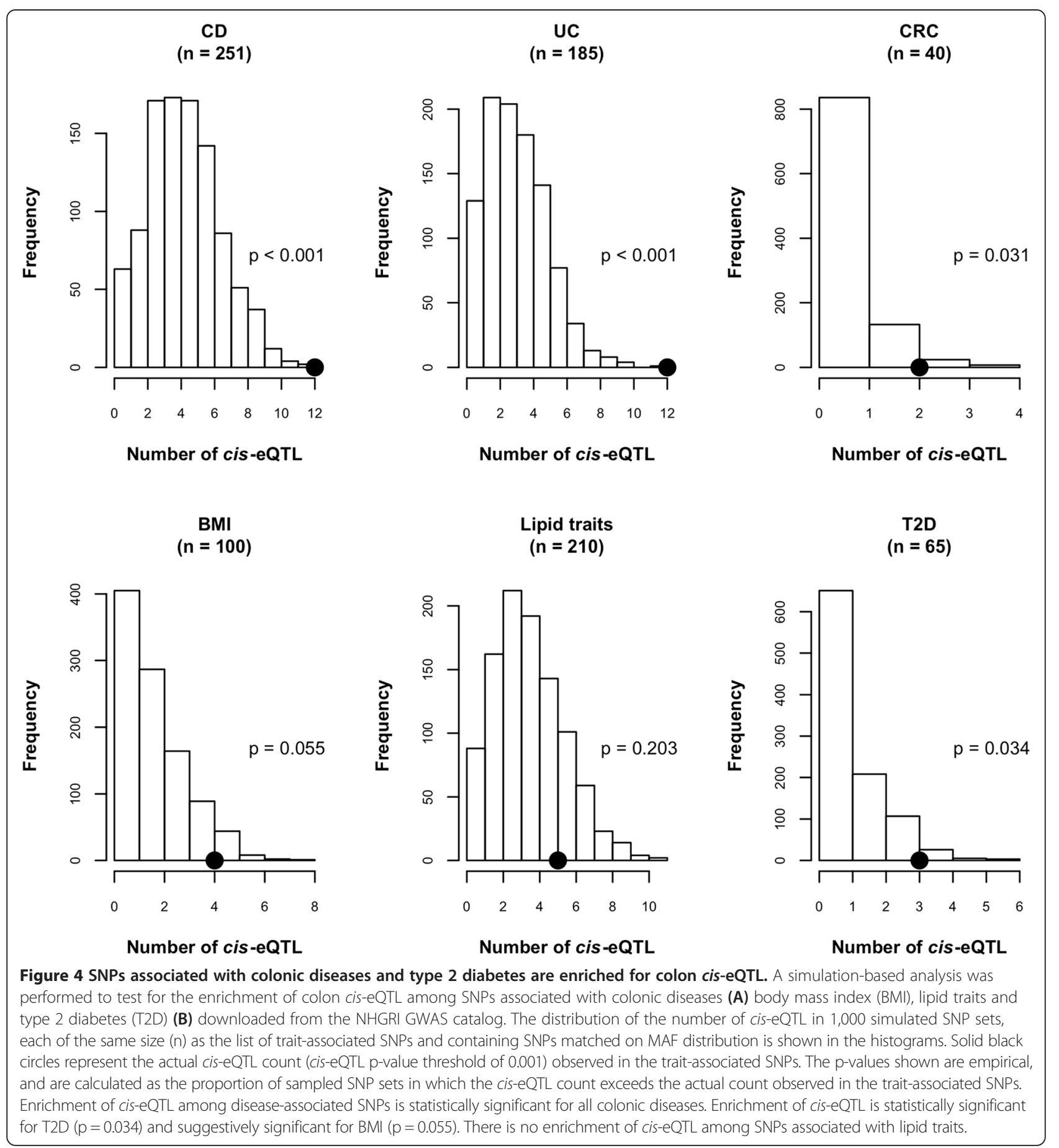

the other traits did not show enrichment for colon eQTL (Additional file 7; Additional file 8: Table S1).

\section{Enrichment of histone marks in colon cis-eQTL}

Simulations were conducted to test whether SNPs overlapping with activating (H3K4me1, H3K4me3, H3K9ac and H3K36me3) and repressing (H3K9me3 and H3K27me3) histone mark peaks were statistically enriched among cis-eQTL at an FDR of 0.10 (see
Methods). Significant enrichment of active histone marks was noted among significant cis-eQTL with the following empirical p-values: $<1.0 \times 10^{-6}(\mathrm{H} 3 \mathrm{~K} 4 \mathrm{me} 1$, H3K4me3 and H3K9ac) and $2.0 \times 10^{-5}$ (H3K36me3) (Figure 5A). There was no significant enrichment for the repressive histone mark H3K9me3 among cis-eQTL $(\mathrm{p}=0.46)$ (Figure 5B). A similar pattern of significant enrichment of active histone marks but not repressive histone marks among colon cis-eQTL was detected in 
Table 4 Enrichment of colon cis-eQTL among NHGRI GWAS SNPs

\begin{tabular}{llll}
\hline Disease or trait & $\begin{array}{l}\text { Number of } \\
\text { GWAS SNPs }\end{array}$ & $\begin{array}{l}\text { Number of GWAS SNPs } \\
\text { that are colon cis-eQTL }\end{array}$ & p-value* \\
\hline CD & 251 & 12 & $<0.001$ \\
UC & 185 & 12 & $<0.001$ \\
CRC & 40 & 2 & 0.031 \\
T2D & 65 & 3 & 0.034 \\
BMI & 100 & 4 & 0.055 \\
\hline
\end{tabular}

*P-values were empirically determined using a simulation-based method in which 1,000 randomized SNP sets, matched in size and MAF distribution to the NHGRI GWAS SNPs, were generated. These simulations yielded a p-value, calculated as the proportion of sampled SNP sets in which the cis-eQTL count exceeds the actual count observed in the GWAS SNPs. For details see Supplementary Methods (Additional file 8). colonic smooth muscle, adipose nuclei, adult liver, and breast myoepithelial cells (data not shown). In human CRC adenocarcinoma cell line (Caco-2), there was enrichment for H3K4me3 $\left(\mathrm{p}<1.0 \times 10^{-6}\right)$ and a suggestive enrichment for H3K36me3 ( $\mathrm{p}=0.060)$, but no significant enrichment for the repressing mark H3K27me3 ( $\mathrm{p}=0.86)$ (Additional file 9). Taken together, these analyses show that colon cis-eQTL are enriched for active histone marks in several tissue types including malignant CRC cell lines.

\section{Discussion}

Gene expression in a disease-relevant tissue is a powerful intermediate phenotype that could help elucidate the functional basis of some risk-associated variants identified in GWAS. Gene expression analysis is especially important as disease-associated variants are enriched in

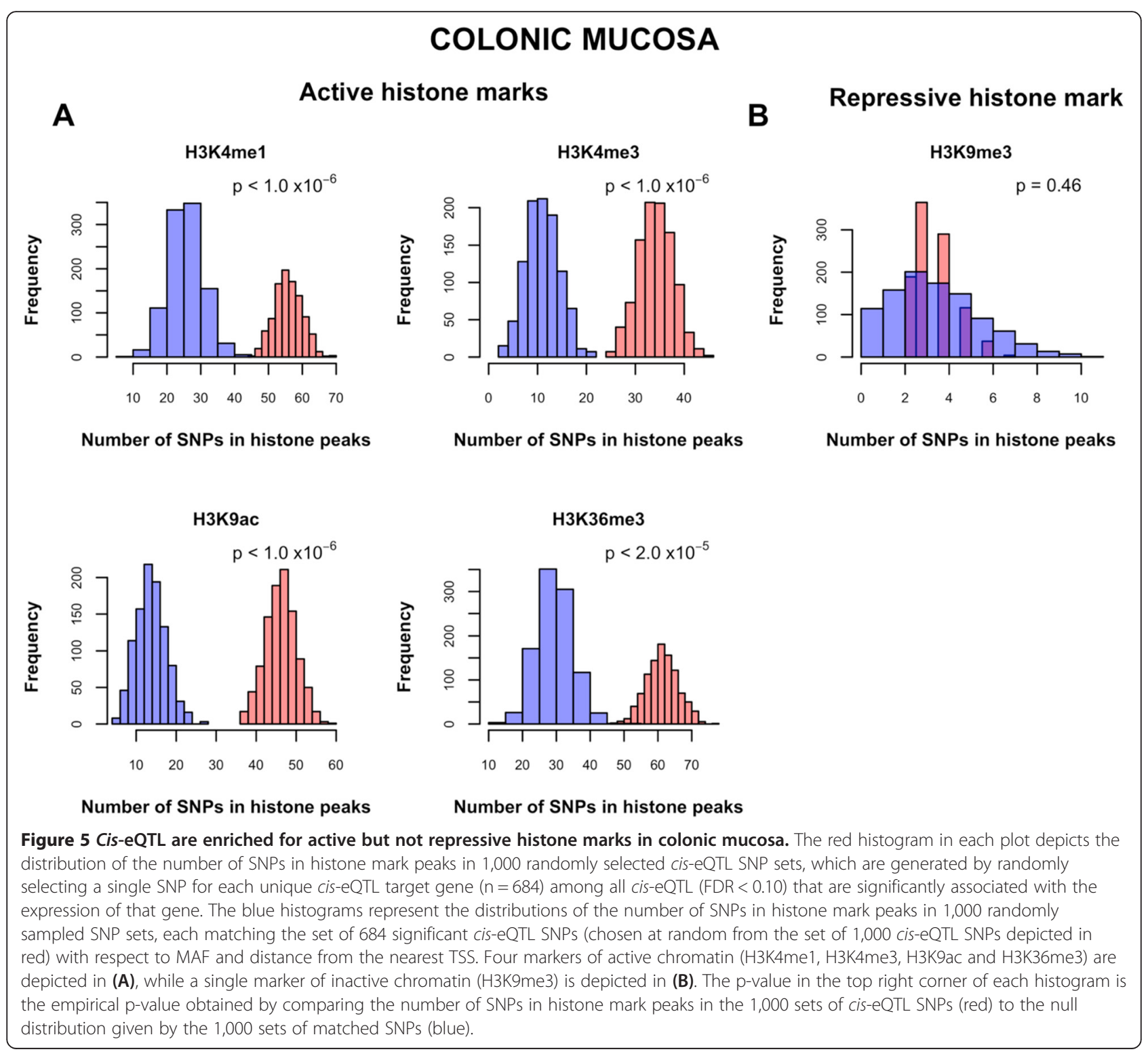


non-coding regions $[33,44,52]$. The analysis of published GWAS variants associated with colonic diseases noted significant enrichment for cis-eQTL in IBD (CD and UC) as well as CRC. Novel target genes for IBDassociated variants were identified and several previously reported eQTL identified in other tissues were validated. Moreover, overlap of colon eQTL in other tissue types, most notably ileum and liver was noted. Colon cis-eQTL were located near TSS as well as enriched for active histone marks and variants with high population differentiation, results that underscore the functional role of eQTL in gene regulation. This analysis could provide insights into the functional consequences of diseaseassociated genetic variants.

Among IBD-associated GWAS SNPs, variants were associated with differences in the expression of endoplasmic reticulum aminopeptidase $2(E R A P 2)$ and scmlike with four MBT domains 1 (SFMBT1) (Figure 2A; Table 2), similar to results in an eQTL mapping study of the human ileum [49]. ERAP2 encodes an endoplasmic reticulum aminopeptidase responsible for major histocompatibility complex class I (MHC1) ligand trimming [53] that has in silico support as a functional variant in CD [10]. SFMBT1 is a polycomb protein with transcriptional repressor activity $[54,55]$ that may regulate a number of genes through epigenetic mechanisms. Additional SNPs for IBD-associated variants identified in this study and their gene targets-rs6545800 with adenylate cyclase (ADCY3), rs4077515 with inositol polyphosphate 5phosphatase (INPP5E), rs9858542 with ubiquitin-like modifier activating enzyme 7 (UBA7), and rs678170 with neuroexophilin and PC-esterase domain family member 1 (NXPE1) and with RNA exonuclease 2 (REXO2) -were novel targets and should be validated in a larger study.

Among CRC-associated GWAS SNPs, rs3802842, was found to be a cis-eQTL in the colon $[26,56]$, a finding that was also recently reported by another study [50]. The target transcript C11orf93 corresponds to an uncharacterized gene known as colorectal cancer associated 2 (COLCA2). A study found that two functional risk variants (rs7130173 and rs10891246), which are in perfect LD with rs3802842, lead to decreased expression of C11orf 93 [57]. These results were similar to our finding that the $C$ allele of rs3802842 also results in decreased expression. While this variant was first identified in individuals of European descent, a previous study by our group validated this SNP as associated with rectal cancer in AA [58], though other groups have not found evidence for an association of rs3802842 with CRC in AA [59]. A recent transethnic GWAS identified an additional SNP (rs79453636) as associated with CRC in AA, independent of rs3802842, that has not been replicated in other populations [60]. Neither rs79453636, nor any SNPs in LD with it, was identified as a colon cis-eQTL in the present study.
Enrichment of colon cis-eQTL among trait-associated variants as shown in Table 4 is consistent with other studies [44,61-63] and adds further evidence for the usefulness of eQTL in improving power to detect significant associations from GWAS. Enrichment of colon cis-eQTL was observed in diseases with colonic involvement (IBD and $\mathrm{CRC}$ ), as well as in T2D and BMI. These results raise the intriguing hypothesis that gene expression in the colon could be functionally linked to diabetes and BMI, though further work is needed to identify specific mechanisms. No significant enrichment was found for colon cis-eQTL among variants associated with autoimmune disorders, cancer or psychiatric disorders. Taken together, these results support the conclusion that eQTL most relevant to disease show clear tissue specificity, highlighting the importance of creating comprehensive eQTL catalogs in diverse tissue types [64].

A substantial proportion of the gene targets of the significant colon cis-eQTL were also found to be cisregulated target genes in other tissue types notably liver and ileum - two tissues closely related to the colon (Table 3). The percentage overlap of cis-eQTL target genes between colon and other tissue types was higher at more stringent FDR thresholds for cis-eQTL associations, which was expected because increased stringency should filter out false positive results. The number of significant target genes shared between studies will also be affected by sample size, significance thresholds and genotyping platforms. Therefore, the actual percentage values of overlap should be regarded as rough estimates of the actual extent of overlap. The high cross-tissue replicability of the colon cis-eQTL is consistent with the observation here of active histone mark enrichment in non-colon tissue types as well as findings from other studies that showed that a large number of cis-eQTL are shared across tissues $[42,46,65]$. The extent to which eQTL have cell-type or context-specificity in the human colon remains to be investigated but could yield important information for further functional characterization.

Consistent with the hypothesis that eQTL have regulatory functions, enrichment was noted for colon cis-eQTL near TSS similar to findings in other tissues $[34,39,46-48]$. Moreover, cis-eQTL were significantly enriched for histone marks associated with active chromatin (H3K4me1, H3K4me3, H3K9ac and H3K36me3) but not with transcriptionally inactive chromatin (H3K9me3 and $\mathrm{H} 3 \mathrm{~K} 27 \mathrm{me} 3$ ) in various tissues and in a CRC cell line. These findings are consistent with the widely accepted theory that cis-eQTL act by affecting transcript stability or the rate of transcript degradation [39,66-68], and other studies that have also noted enrichment of eQTL among activating cis-elements but not in regions of repressed chromatin [69-71]. 
A substantial proportion of colon cis-eQTL in this study were highly differentiated between European and African populations $\left(\mathrm{F}_{\mathrm{ST}}>0.25\right)$ as shown in Figure 3. The degree of population differentiation among these eQTL suggests that they may well reflect local adaptation to environment. A similar finding was previously described for LCL eQTL [72]. It remains to be investigated whether such colon eQTL, which exhibit large allele frequency differences between European and African populations, contribute to inter-ethnic gene expression differences in the colon that translate into differences in disease risk. Integration of GWAS results with population genetic approaches may prove instrumental in understanding the genetic basis of differences in IBD and CRC risk between Europeans and AA [73-76].

A limitation of this study is the moderate sample size, which reduces power to detect significant eQTL associations (especially those acting in trans), thereby resulting in a high false negative rate. Further investigation in a larger cohort is likely to result in more significant eQTL associations, and is warranted to confirm findings from this study. Another caveat of this study is that the GWAS SNPs used in the analyses were identified in Europeans, whereas the eQTL were identified in AA. Although many of the variants identified through GWAS in European populations generalize to other populations, there are differences in genetic susceptibility between populations, due to several factors including epistasis, gene-environment interactions, population-specific polymorphisms and differential LD patterns [77]. Therefore, it is possible that there are differences in genetic associations between European and AA populations. As IBD and CRC GWAS in populations of African descent become available, the overlap between eQTL identified in this study and GWAS SNPs in these populations should be investigated.

\section{Conclusions}

In the present study, a comprehensive map of eQTL in the healthy colon of AA was generated as a resource for genetic studies of colonic diseases. The analysis showing the enrichment of colon eQTL among SNPs associated with colonic diseases supported the usefulness of colon eQTL as a tissue-specific tool to improve understanding of colonic disease susceptibility. The utility of colon eQTL for studying the genetic basis of inter-ethnic differences in colonic disease risk was demonstrated by showing their enrichment for SNPs that exhibit high allele frequency differences between European and African populations. These SNPs could mediate populationspecific gene expression responses, which could translate into differences in disease risk.

This study offers novel insights into the functional basis of genetic susceptibility for colonic inflammatory and malignant diseases and provides a tissue-specific resource for future studies. Characterization of the genetic architecture of gene regulation in the human colon informs the functional impact of GWAS variants and could benefit understanding of the biological differences in colonic disease between different ethnic groups. The findings in this study demonstrate that eQTL are important in the susceptibility to inflammatory and malignant diseases of the colon, underscoring the utility of eQTL mapping for elucidating the genetics and biology underlying colonic diseases. Furthermore the eQTL map presented here could benefit understanding of the biological differences in colonic disease between different ethnic groups. This is especially important given that there is a paucity of genetic studies of colonic diseases in AA populations and our understanding of the genetics and etiology of these diseases is based on Europeans, which may not be applicable to other populations. Further efforts should be made to intersect our eQTL data with IBD and CRC genetic studies, for a better understanding of the genetic mechanisms and inter-ethnic differences in these diseases.

\section{Methods}

\section{Subjects}

A total of 48 AA subjects were included. All subjects underwent colonoscopy at the University of Illinois Chicago for screening purposes. Blood samples were obtained at the time of colonoscopy. Colonic biopsies were obtained using standard forceps (Boston Scientific, Natick, MA) in all subjects at $20 \mathrm{~cm}$ from the anal verge at the recto-sigmoid junction. Biopsies were placed immediately into RNAlater (Life Technologies Corporation, Grand Island, NY), and cryopreserved according to the manufacturer's instructions. Subjects' mean age (standard deviation) was 59.0 years (8.5). While the intention was to include only males in order to reduce heterogeneity, two female subjects were included due to sample mislabeling. Written informed consent was obtained from participants.

\section{Ethics statement}

This research was approved by the Institutional Review Boards at the University of Illinois at Chicago and the University of Chicago.

\section{Genotyping and imputation}

Colon samples from study subjects were genotyped on the Affymetrix Axiom Genome-Wide Pan-African array that includes 2,217,402 probes (Affymetrix, Santa Clara, CA). Genotypes were called using the Illumina Genome Studio software package (Illumina, San Diego, CA). QC filters applied to the data are outlined in Figure 1. The analysis was limited to SNPs that map to unique chromosomal locations based on the latest annotation 
file for the genotyping array (Release 33). Markers with $<95 \%$ genotype call rates, MAF $<0.05$ or HWE $\mathrm{p}<10^{-6}$ were excluded from analysis. Markers with HWE $\mathrm{p}<10^{-4}$ for which there were no heterozygotes or homozygotes for the minor allele, as well as markers with HWE $\mathrm{p}<10^{-3}$ for which there were $<6$ heterozygotes or homozygotes for the minor allele were also excluded. Finally, all non-autosomal SNPs were omitted. The final genotyped dataset contained $1,492,955$ autosomal SNPs in 48 subjects with an average call rate of $99.69 \%$.

Imputation was performed with IMPUTE2 version 2.2.2 [78,79] using the 1000 Genomes Phase I integrated variant set release (v3) (https://mathgen.stats.ox.ac.uk/ impute/impute_v2.html) [51] as reference panel. IMPUTE2 uses a reference panel of known haplotypes and a fine-scale recombination map to infer missing genotypes in a study dataset that has been typed at a relatively sparse set of markers. IMPUTE2 assigns each imputed SNP an INFO score on the basis of imputation quality. INFO scores can take values from 0 to 1 , where values closer to 1 indicate that there is little uncertainty in the imputed genotype. Imputed SNPs with MAF $<0.05$ and IMPUTE2-info score $<0.5$ were excluded from the analysis, leaving 8,400,922 imputed SNPs in 48 subjects. Imputed genotypes were coded as allelic dosages (i.e. estimated fractional counts of the non-reference allele ranging continuously from 0 to 2 ).

\section{Transcriptional profiling}

RNA was extracted from manually ground tissue using the Maxwell 16 Tissue LEV Total RNA Purification Kit (Promega, Fitchburg, WI) for automated purification on the Maxwell 16 Instrument (Promega, Fitchburg, WI). Gene expression levels were measured using the Illumina HT-12v4 Expression BeadChip whole-genome expression array containing 47,231 gene probes (Illumina, San Diego, CA), according to manufacturer's instructions. The Illumina GenomeStudio software was used to extract the signal intensity for each probe, which were then further preprocessed using the lumi package of Bioconductor (http://www.bioconductor.com) $[80,81]$ in $\mathrm{R}$ version 3.0.2. The preprocessing included background correction of the expression data, followed by variance stabilization transformation (VST), log2-transformation and quantile-normalization.

BLAST-like alignment tool (BLAT) pairwise sequence alignment algorithm was used to map the probe sequences onto the RefSeq transcriptome (downloaded from the UCSC Genome Browser: http://hgdownload. cse.ucsc.edu/goldenPath/hg19/bigZips). All probes mapping to multiple Entrez Gene IDs were omitted and only probes that map uniquely to autosomal genes with no mismatches were included. Probes that encompass a common SNP $(\mathrm{MAF} \geq 0.05)$ in either 1000 Genomes AFR or EUR populations were further excluded, leaving 25,610 probes with unique perfect matches to autosomal genes that do not contain common SNPs. Furthermore only probes with an Illumina detection $\mathrm{p}<0.01$ in at least one individual were included in further analysis.

Eight individuals were identified as outliers based their poor clustering with other subjects on gene expression heat map (Additional file 2). There was no correlation between any of the demographic and clinical characteristics and outlier status. The separation of the outliers from the rest of the samples seemed to be driven by systematic differences in gene expression rather than by expression differences of only a few genes. Four of the outlier samples had low RNA integrity numbers (RIN) $(<8)$. As the outliers with RIN $>8$ seemed to cluster with the four outliers with the low RIN scores, the outliers were likely driven by poor RNA quality. Upon the exclusion of the eight outlier samples, there were no further outliers on the gene expression heat map. The outliers were excluded from the gene expression analysis prior to the microarray pre-processing steps in Bioconductor. After the removing probes that were not significantly expressed, the final expression dataset included 16,252 probes interrogating 12,363 unique genes in 40 subjects. The gene expression data is available at Gene Expression Omnibus (http://www.ncbi.nlm.nih.gov/projects/geo) with the accession number GSE 56789.

The linear model that is used in the eQTL analysis assumes that the expression values for each probe follow a normal distribution, and it may be sensitive to the presence of outliers and non-normality. Thus the log2transformed and quantile-normalized expression values for each probe were transformed into a standard normal distribution using the rank-based inverse normal transformation implemented in the qqnorm function in $\mathrm{R}$ prior to the eQTL analysis.

Colon biopsies were composed of primarily epithelial cells with a small amount of stromal cells. At least 2 biopsies from the colon were used per individual to minimize tissue heterogeneity. Overall, expression of an epithelial marker E-cadherin ( $C D H 1)$ did not show significant interindividual variation when normalized to a housekeeping gene GAPDH across the 40 individuals (data not shown).

\section{Ancestry estimates}

PCA of the genotype data was used to quantify the proportions of European and African ancestry in the AA subjects. Samples from HapMap ASW, CEU, and YRI populations were used as reference populations. PCA analysis was performed in EIGENSTRAT [82] using an LD-pruned dataset of 48,553 SNPs. The first PC from the analysis was included as a covariate to account for ancestry in subsequent analyses. 


\section{eQTL mapping}

eQTL mapping was performed using the Matrix eQTL $R$ package [83]. Associations between SNP genotype and probe expression level were analyzed using a linear regression model with additive genotype effects. Cis-eQTL were defined as associations between SNPs within 1 mega base pair $(\mathrm{Mb})$ of the TSS or transcription end site (TES) of a gene. Trans-eQTL were defined as association signals from SNPs located greater than $1 \mathrm{Mb}$ from the TSS and TES of a gene or on a different chromosome. FDR calculations were performed separately for cis- and trans-eQTL in Matrix eQTL according to the Benjamini and Hochberg method [84]. A PCA-based approach was used to correct for confounding variability due to hidden factors in the gene expression data [85] as outlined in the Supplementary Methods (Additional file 8).

To determine the number of independent cis-eQTL associations at the different FDR thresholds, a stepwise association model was applied that involves repeating the eQTL association analysis conditioned on the most significant cis-eQTL for each gene expression probe, until there were no more significant cis-associations left as described in the Supplementary Methods (Additional file 8 ).

\section{Colon cis-eQTL associated with colonic diseases}

All NHGRI GWAS SNPs associated with colonic diseases $(\mathrm{CD}, \mathrm{UC}$ and $\mathrm{CRC})$ that were either significant cis-eQTL $($ FDR $<0.10)$ or in high LD with a cis-eQTL were identified. All SNPs in high LD $\left(r^{2} \geq 0.8\right)$ with the cis-eQTL were obtained from SNAP database (http:// www.broadinstitute.org/mpg/snap) [86] using CEU as the reference. Associations were visualized using box plots that show expression as a function of eQTL genotype. For details, refer to Supplementary Methods (Additional file 8).

\section{Evaluation of cis-eQTL enrichment among trait-associated SNPs}

The enrichment of significant cis-eQTL among SNPs associated with various complex traits and diseases were investigated. For the enrichment analyses, only traitassociated SNPs that were among the 8,400,922 SNPs analyzed were considered. Trait-associated GWAS SNPs were downloaded from the NHGRI Catalog of Published GWAS using the default p-value threshold of $10^{-5}$ [87]. SNPs that were not identified in populations of European descent were excluded. The complex traits/diseases were classified into 5 broad categories: (1) autoimmune disorders (celiac disease $[n=57]$, psoriasis $[n=35]$ and RA [ $\mathrm{n}=54]$ ) (2) cancers (breast cancer $[\mathrm{n}=100]$, prostate cancer $[\mathrm{n}=121]$ and melanoma $[\mathrm{n}=16]),(3)$ colonic diseases $(C D[n=251], U C[n=185]$ and CRC $[n=40])$,
(4) BMI $(\mathrm{n}=100)$, lipid traits $(\mathrm{n}=210)$ and T2D $(\mathrm{n}=$ 65) and (5) psychiatric disorders (ADHD [n $=179]$, BD $[n=257]$ and schizophrenia $[n=247])$. The number of GWAS SNPs for each trait that fit the aforementioned criteria and were thus included in the enrichment analyses are indicated by $\mathrm{n}$ and listed inside the brackets.

Simulation-based enrichment analyses were conducted as previously described [44], to evaluate if the traitassociated SNPs were enriched for colon cis-eQTL. Briefly, 1,000 randomized SNP sets were generated sampled without replacement from the 8,400,922 study SNPs. Since there was an overrepresentation of higherfrequency variants among the significant cis-eQTL compared to all study SNPs (Additional file 10), SNPs were matched on MAF distribution. SNPs were also matched on distance to the nearest TSS since cis-eQTL were more enriched around TSS compared to other SNPs (Additional file 11). For each sampled SNP set and the set of NHGRI SNPs, the number of SNPs that were also cis-eQTL was determined. The relatively relaxed significance threshold of $\mathrm{p}<0.001$ was used for eQTL $\mathrm{p}$-values since the numbers of trait-associated cis-eQTL were too low to quantify enrichment at higher thresholds of significance. An empirical p-value for cis-eQTL enrichment was obtained based on the simulations, calculated as the proportion of sampled SNP sets in which the cis-eQTL count exceeds the actual cis-eQTL count observed in the NHGRI SNPs.

\section{Bioinformatic analysis of cis-eQTL}

The genomic locations of cis-eQTL were characterized by plotting their position relative to the TSS of genes. The enrichment of histone marks among significant ciseQTL at an FDR threshold of 0.10 was evaluated using a modified version of the simulation-based strategy that was used to test for eQTL enrichment among traitassociated SNPs. Briefly, the number of SNPs in histone marks peaks among 1,000 sets of independent cis-eQTL were compared to that among 1,000 randomized SNP sets, matched based on the distributions of MAF and distance from the nearest TSS to the independent ciseQTL. For details and adaptations, see Supplementary Methods (Additional file 8).

\section{Comparative analysis with cis-eQTL from other tissues}

The overlap between cis-eQTL in the colon and cis-eQTL in other tissue was determined using the Genotype-Tissue Expression (GTEx) eQTL browser (available at http://www. ncbi.nlm.nih.gov/gtex/GTEX2/gtex.cgi), which allows the query of eQTL results from multiple previous studies by SNP or gene name. To evaluate the overlap of the colon cis-eQTL with cis-eQTL from another tissue, the GTEx eQTL browser was queried to obtain cis-eQTL p-values for 
genes regulated by a significant colon cis-eQTL identified in this study (at four different FDR thresholds: 0.01, 0.05, 0.10 and 0.20 ). The number of genes that were also cis-regulated, defined as within $1 \mathrm{Mb}$ of a gene, in other studies was determined at a p-value cut-off of $10^{-5}$. These studies included the following tissue types: LCLs [37], liver [35] and brain [48]. Cis-eQTL target genes were considered to be overlapping between the colon and another tissue if both studies had at least one cis-eQTL for that target gene. Overlap of colon with skin cis-eQTL (defined as within $1 \mathrm{Mb}$ of a gene and with $\mathrm{p}<10^{-5}$ ) [46] and colon with ileum cis-eQTL (defined as within 50 kilo base pairs $(\mathrm{kb})$ of a gene and with $\mathrm{p}<10^{-5}$ ) [49] was also investigated using the same approach. Skin ciseQTL data was obtained from the University of Michigan, Center for Statistical Genetics website (http://www.sph. umich.edu/csg/junding/eQTL/). Ileum cis-eQTL data was downloaded from the Additional file of the article [49]. For each tissue type, the total number of target genes that overlap between colon and other tissues was reported along with the number of overlapping target genes that are associated with the same cis-eQTL SNP (or a proxy with $r^{2} \geq 0.8$ ) in both tissues. The number of SNP-gene pairs that overlap between the present study and other eQTL studies was reported as well. Our rationale for focusing on the overlap of eQTL target genes rather than the overlap of eQTL SNPs, is that genes are more comparable and less biased across different studies that use different genotyping platforms with different marker sets.

\section{Fixation index analysis of cis-eQTL}

To estimate the level of genetic differentiation between European and African populations at the colon cis-eQTL, $\mathrm{F}_{\mathrm{ST}}$ values for all colon cis-eQTL $(\mathrm{n}=14,177$; FDR $<0.20)$ were calculated by using Weir and Cockerham's unbiased estimator [88]. For the $\mathrm{F}_{\mathrm{ST}}$ calculations, genotype data for EUR and AFR populations from the 1000 Genomes Project was used [54]. To test whether the number of independent colon cis-eQTL that show high population differentiation between European and African populations is greater than that expected by chance, we performed a simulation-based analysis which we describe in detail in the Supplementary Methods (Additional file 8). SNPs that show high population differentiation between EUR and AFR were defined as those with $\mathrm{F}_{\mathrm{ST}}>0.25$. The number of highly population differentiated SNPs among all 1,000 randomized SNP sets was compared to the number of highly population differentiated SNPs among the sets of independent cis-eQTL using the MannWhitney test. High $\mathrm{F}_{\mathrm{ST}} \mathrm{SNPs}\left(\mathrm{F}_{\mathrm{ST}}>0.10\right)$ were identified among cis-eQTL that were associated with $\mathrm{CD}$, UC or $\mathrm{CRC}$ and allele frequency plots showing the worldwide distributions of ancestral and derived alleles in human populations were constructed. The data used in the generation of these plots are described in detail in a previous publication [89].

\section{Additional files}

Additional file 1: Figure S1. PCA plot of the genotype data from the study samples along with HapMap reference populations. The 48 African American subjects cluster with HapMap African American samples on the principal components analysis (PCA) plot, indicating mixed African and European ancestry. A PCA-based analysis was performed in EIGENSTRAT to estimate the proportions of European and African ancestry for each subject (represented by PC1). HapMap populations of African ancestry in Southwest USA (ASW), Utah residents of Northern and Western European ancestry (CEU) and Yoruba in Ibadan, Nigeria (YRI) were used as references in the PCA plot and are color-coded as indicated in the legend. PC1 was included as a covariate in the eQTL analysis to account for ancestry.

Additional file 2: Figure S2. Heat map visualization and hierarchical clustering of the gene expression data. Study samples are organized based on the similarity of log2-transformed and quantile-normalized gene expression levels across all probes evaluated in the study. The heat maps represent the pairwise correlation matrix of similarity among the study subjects across all probes. Each row and column represents one of the subjects. The color scale indicates the degree of correlation from lowest (white) to highest (red). (A) Heat map corresponding to the gene expression data for the 48 subjects prior to the removal of outliers. The first eight subjects from the left side of the plot were deemed as outliers and excluded from the eQTL analysis. (B) Heat map corresponding to the gene expression data for the 40 remaining subjects after the removal of outliers.

Additional file 3: Figure S3. The numbers of significant cis-eQTL probes were maximized with the inclusion of the first five PCs of the gene expression data as covariates. Each of the first 20 PCs from the gene expression data were sequentially included as covariates in the analysis and the numbers of gene expression probes that were associated with a cis-eQTL were determined for each at two different FDR thresholds for SNP-gene associations. The maximum numbers of gene expression probes associated with a cis-eQTL were obtained when the first five PCs from the gene expression data were used as covariates in the eQTL analysis. Thus the first five PCs were included as covariates in subsequent analyses, to correct for unmeasured variation in the gene expression data.

Additional file 4: Figure S4. The colon eQTL dataset is enriched for cis- but not trans-eQTL. (A) The Q-Q plot shows the eQTL association p-values for all SNP-probe pairs (8,400,922 SNPs $\times 16,252$ probes) evaluated in the study. Red dots represent cis-and blue dots represent trans-association $p$-values. The gray line is the identity line $(y=x)$ representing the null distribution under which there is no association between SNP genotype and probe expression level. Cis-association p-values deviate strongly from the identity line, demonstrating an enrichment of significant associations. There is no such enrichment for trans-associations. (B) The histogram shows the eQTL association p-values for all cis SNP-probe pairs. There is a clear enrichment of cis-associations with small p-values.

Additional file 5: Figure S5. Cis-eQTL cluster roughly symmetrically around TSS. The scatter plot depicts the distribution of cis-eQTL relative to TSS. Each dot represents the most significant cis-associated SNP for each gene expression probe. - $\log 10$ (p-value) for the SNP-probe association (y-axis) is plotted against the base pair (bp) distance of the associated SNP from the TSS of the transcript that the probe is interrogating ( $x$-axis). Negative and positive values of the distance denote SNPs $5^{\prime}$ and $3^{\prime}$ of TSS (set at 0), respectively. The majority of significant cis-eQTL are found within $100 \mathrm{~kb}$ of TSS.

Additional file 6: Figure S6. Human populations across the world differ in the designations of ancestral and derived alleles as major alleles for UC-associated colon cis-eQTL rs9847710. rs9847710 exhibits a relatively high level of population differentiation between 1000 Genomes EUR and AFR populations $\left(F_{S T}>0.10\right)$. The plot shows the distribution of $r s 9847710$ alleles in multiple populations worldwide. The haplotype frequencies for 
different human populations are depicted in pie charts where the ancestral allele (C) is shown in blue and the derived allele (T) is shown in orange.

Additional file 7: Figure S7. SNPs associated with diseases that do not involve the colon are not enriched for colon cis-eQTL. This is similar to Figure 4, except the plots depict the enrichment of colon cis-eQTL among SNPs associated with (A) autoimmune disorders, (B) cancers and (C) psychiatric disorders. There is no significant enrichment for colon cis-eQTL among SNPs associated with these disorders.

\section{Additional file 8: Supplementary Methods.}

Additional file 9: Figure S8. Cis-eQTL are enriched for active but not repressive histone marks in a colorectal adenocarcinoma cell line. This is similar to Figure 5, except the histone mark data is from a colorectal adenocarcinoma cell line (Caco-2) and only two active histone marks (H3K4me3 and H3K36me3) and one repressive histone mark (H3K27me3) are depicted. Colon cis-eQTL are enriched for active histone marks with the enrichment reaching statistical significance for H3K4me3 $\left(p<1.0 \times 10^{-6}\right)$ and being highly suggestive for H3K36me3 $(p=0.060)$. There is no statistically significant enrichment of the repressive histone mark H3K27me3.

Additional file 10: Figure S9. MAF distribution of colon cis-eQTL is skewed towards higher frequencies compared with the complete set of study SNPs. MAF distribution of the complete set of study SNPs (top graph) is presented, along with the MAF distributions of cis-eQTL at four different FDR thresholds. The skew towards higher frequencies becomes more pronounced as the FDR threshold becomes more stringent.

Additional file 11: Figure S10. Colon cis-eQTL are closer to TSS than other SNPs. The histograms show the distributions of the base pair (bp) distances to the nearest TSS for all study SNPs that are within 1 mega base pair (Mb) of a TSS and significant cis-eQTL at various FDR thresholds. Negative distances refer to SNPs upstream ( $5^{\prime}$ ) of the TSS (set at 0 ) while positive distances refer to those downstream (3').

\section{Abbreviations}

AA: African American; ADCY3: Adenylate cyclase 3; ADHD: Attention deficit hyperactivity disorder; AFR: 1000 Genomes Project African population; ASW: African ancestry in Southwest USA; BD: Bipolar disorder; BLAT: BLAST-like alignment tool; BMI: Body mass index; CD: Crohn's disease; CEU: Utah residents with Northern and Western European ancestry; ChIP: Chromatin immunoprecipitation; COLCA2: Colorectal cancer associated 2; CRC: Colorectal cancer; eQTL: Expression quantitative trait loci; ERAP2: Endoplasmic reticulum aminopeptidase 2; EUR: 1000 Genomes Project European population; FDR: False discovery rate; Fst: Fixation index; GEO: Gene Expression Omnibus; GTEx: Genotype-Tissue Expression; GWAS: Genome-wide association study; HWE: Hardy-Weinberg equilibrium; IBD: Inflammatory bowel disease; INPP5E: Inositol polyphosphate-5-phospatase; Kb: Kilo base pair; LCL: Lymphoblastoid cell line; LD: Linkage disequilibrium; MAF: Minor allele frequency; Mb: Mega base pair; MHC1: Major histocompatibility complex class l; mRNA: Messenger ribonucleic acid; NHGRI: National Human Genome Research Institute; NXPE1: Neuroexophilin and PC-esterase domain family member 1; PC: Principal component; PCA: Principal components analysis; QC: Quality control; Q-Q: Quantile-quantile; RA: Rheumatoid arthritis; RIN: RNA integrity number; REXO2: RNA exonuclease 2; SFMBT1: Scm-like with four MBT domains 1; SNP: Single nucleotide polymorphism; T2D: Type 2 diabetes; TES: Transcription end site; TSS: Transcription start site; UBA7: Ubiquitin-like modifier activating enzyme 7; UC: Ulcerative colitis; VST: Variance stabilizing transformation; YRI: Yoruba in Ibadan, Nigeria.

\section{Competing interests}

The authors declare that they have no competing interests.

\section{Authors' contributions}

NAE and SSK designed the study, and obtained funding. RMX and XL collected and processed the colon samples, and performed the genotyping and gene expression profiling. $\mathrm{IH}$ analyzed and interpreted the data, and created the figures. ADS, KO, NAE and SSK provided critical input into data analysis and interpretation. E.R.G. performed the fixation index analyses. SSK monitored the study conduct. IH and SSK wrote the manuscript, with input and review from ERG, ADS, KO and NAE. All authors read and approved the final draft of the manuscript.

\section{Acknowledgments}

We thank Joseph Maranville for critical review of the manuscript and Barbara Stranger for helpful discussions. This work was supported by the National Institutes of Health (R01CA 140804 and U01CA 153060 to NAE, K08CA 142892 to SSK) and the American Cancer Society Illinois Division (223187) to XL.

\section{Author details}

'Committee on Genetics, Genomics and Systems Biology, Chicago, IL 60637, USA. ${ }^{2}$ Department of Medicine, 900 East 57th Street, MB\#9, Chicago, IL 60637, USA. ${ }^{3}$ Department of Pediatrics, University of Chicago, Chicago, IL 60637, USA. ${ }^{4}$ Department of Medicine, Yale University, New Haven, CT 06510, USA. ${ }^{5}$ University of Arizona Cancer Center, Tucson, AZ 85724, USA. ${ }^{6}$ Division of Genetic Medicine, Department of Medicine, Vanderbilt University, Nashville, TN 37232, USA.

Received: 3 September 2014 Accepted: 29 January 2015 Published online: 27 February 2015

\section{References}

1. Anderson CA, Boucher G, Lees CW, Franke A, D'Amato M, Taylor KD, et al. Meta-analysis identifies 29 additional ulcerative colitis risk loci, increasing the number of confirmed associations to 47. Nat Genet. 2011;43(3):246-52.

2. Franke A, Balschun T, Sina C, Ellinghaus D, Hasler R, Mayr G, et al. Genome-wide association study for ulcerative colitis identifies risk loci at 7q22 and 22q13 (IL17REL). Nat Genet. 2010;42(4):292-4.

3. Franke A, Balschun T, Karlsen TH, Hedderich J, May S, Lu T, et al. Replication of signals from recent studies of Crohn's disease identifies previously unknown disease loci for ulcerative colitis. Nat Genet. 2008;40(6):713-5.

4. McGovern DP, Gardet A, Torkvist L, Goyette P, Essers J, Taylor KD, et al. Genome-wide association identifies multiple ulcerative colitis susceptibility loci. Nat Genet. 2010;42(4):332-7.

5. Barrett JC, Lee JC, Lees CW, Prescott NJ, Anderson CA, Phillips A, et al. Genome-wide association study of ulcerative colitis identifies three new susceptibility loci, including the HNF4A region. Nat Genet. 2009;41(12):1330-4.

6. Silverberg MS, Cho JH, Rioux JD, McGovern DP, Wu J, Annese V, et al. Ulcerative colitis-risk loci on chromosomes 1p36 and 12q15 found by genome-wide association study. Nat Genet. 2009;41(2):216-20.

7. Julia A, Domenech E, Ricart E, Tortosa R, Garcia-Sanchez V, Gisbert JP, et al. A genome-wide association study on a southern European population identifies a new Crohn's disease susceptibility locus at RBX1-EP300. Gut. 2013;62(10):1440-5.

8. Kenny EE, Pe'er I, Karban A, Ozelius L, Mitchell AA, Ng SM, et al. A genome-wide scan of Ashkenazi Jewish Crohn's disease suggests novel susceptibility loci. PLoS Genet. 2012;8(3):e1002559.

9. Huang J, Ellinghaus D, Franke A, Howie B, Li Y. 1000 Genomes-based imputation identifies novel and refined associations for the Wellcome Trust Case Control Consortium phase 1 Data. Eur J Hum Genet. 2012;20(7):801-5.

10. Franke A, McGovern DP, Barrett JC, Wang K, Radford-Smith GL, Ahmad T, et al. Genome-wide meta-analysis increases to 71 the number of confirmed Crohn's disease susceptibility loci. Nat Genet. 2010;42(12):1118-25.

11. McGovern DP, Jones MR, Taylor KD, Marciante K, Yan X, Dubinsky M, et al. Fucosyltransferase 2 (FUT2) non-secretor status is associated with Crohn's disease. Hum Mol Genet. 2010;19(17):3468-76.

12. Barrett JC, Hansoul S, Nicolae DL, Cho JH, Duerr RH, Rioux JD, et al. Genome-wide association defines more than 30 distinct susceptibility loci for Crohn's disease. Nat Genet. 2008;40(8):955-62.

13. Raelson JV, Little RD, Ruether A, Fournier $H$, Paquin B, Van Eerdewegh $P$, et al. Genome-wide association study for Crohn's disease in the Quebec Founder Population identifies multiple validated disease loci. Proc Natl Acad Sci U S A. 2007;104(37):14747-52.

14. Franke A, Hampe J, Rosenstiel P, Becker C, Wagner F, Hasler R, et al. Systematic association mapping identifies NELL1 as a novel IBD disease gene. PLoS One. 2007;2(8):e691.

15. The Wellcome Trust Case Control Consortium. Genome-wide association study of 14,000 cases of seven common diseases and 3,000 shared controls. Nature. 2007;447(7145):661-78.

16. Parkes M, Barrett JC, Prescott NJ, Tremelling M, Anderson CA, Fisher SA, et al. Sequence variants in the autophagy gene IRGM and multiple other replicating loci contribute to Crohn's disease susceptibility. Nat Genet. 2007;39(7):830-2. 
17. Rioux JD, Xavier RJ, Taylor KD, Silverberg MS, Goyette P, Huett A, et al. Genome-wide association study identifies new susceptibility loci for Crohn disease and implicates autophagy in disease pathogenesis. Nat Genet. 2007;39(5):596-604.

18. Libioulle C, Louis E, Hansoul S, Sandor C, Farnir F, Franchimont D, et al. Novel Crohn disease locus identified by genome-wide association maps to a gene desert on 5 p13.1 and modulates expression of PTGER4. PLoS Genet. 2007;3(4):e58.

19. Festen EA, Goyette P, Green T, Boucher G, Beauchamp C, Trynka G, et al. A meta-analysis of genome-wide association scans identifies IL18RAP, PTPN2, TAGAP, and PUS10 as shared risk loci for Crohn's disease and celiac disease. PLoS Genet. 2011;7(1):e1001283.

20. Fernandez-Rozadilla C, Cazier JB, Tomlinson IP, Carvajal-Carmona LG, Palles C, Lamas MJ, et al. A colorectal cancer genome-wide association study in a Spanish cohort identifies two variants associated with colorectal cancer risk at 1p33 and 8p12. BMC Genomics. 2013;14:55.

21. Peters U, Jiao S, Schumacher FR, Hutter CM, Aragaki AK, Baron JA, et al. Identification of genetic susceptibility loci for colorectal tumors in a genome-wide meta-analysis. Gastroenterology. 2013;144(4):799-807. e724.

22. Dunlop MG, Dobbins SE, Farrington SM, Jones AM, Palles C, Whiffin N, et al. Common variation near CDKN1A, POLD3 and SHROOM2 influences colorectal cancer risk. Nat Genet. 2012;44(7):770-6.

23. Peters U, Hutter CM, Hsu L, Schumacher FR, Conti DV, Carlson CS, et al. Meta-analysis of new genome-wide association studies of colorectal cancer risk. Hum Genet. 2012;131(2):217-34.

24. Houlston RS, Cheadle J, Dobbins SE, Tenesa A, Jones AM, Howarth K, et al. Meta-analysis of three genome-wide association studies identifies susceptibility loci for colorectal cancer at 1q41, 3q26.2, 12q13.13 and 20q13.33. Nat Genet. 2010;42(11):973-7.

25. Houlston RS, Webb E, Broderick P, Pittman AM, Di Bernardo MC, Lubbe S, et al. Meta-analysis of genome-wide association data identifies four new susceptibility loci for colorectal cancer. Nat Genet. 2008;40(12):1426-35.

26. Tenesa A, Farrington SM, Prendergast JG, Porteous ME, Walker M, Haq N, et al. Genome-wide association scan identifies a colorectal cancer susceptibility locus on 11q23 and replicates risk loci at 8q24 and 18q21. Nat Genet. 2008:40(5):631-7.

27. Tomlinson IP, Webb E, Carvajal-Carmona L, Broderick P, Howarth K, Pittman AM, et al. A genome-wide association study identifies colorectal cancer susceptibility loci on chromosomes 10p14 and 8q23.3. Nat Genet. 2008;40(5):623-30.

28. Broderick P, Carvajal-Carmona L, Pittman AM, Webb E, Howarth K, Rowan A, et al. A genome-wide association study shows that common alleles of SMAD7 influence colorectal cancer risk. Nat Genet. 2007;39(11):1315-7.

29. Tomlinson I, Webb E, Carvajal-Carmona L, Broderick P, Kemp Z, Spain S, et al. A genome-wide association scan of tag SNPs identifies a susceptibility variant for colorectal cancer at 8q24.21. Nat Genet. 2007;39(8):984-8.

30. Zanke BW, Greenwood CM, Rangrej J, Kustra R, Tenesa A, Farrington SM, et al. Genome-wide association scan identifies a colorectal cancer susceptibility locus on chromosome 8q24. Nat Genet. 2007;39(8):989-94.

31. Altshuler D, Daly MJ, Lander ES. Genetic mapping in human disease. Science. 2008;322(5903):881-8.

32. Edwards SL, Beesley J, French JD, Dunning AM. Beyond GWASs: illuminating the dark road from association to function. Am J Hum Genet. 2013;93(5):779-97.

33. Cookson W, Liang L, Abecasis G, Moffatt M, Lathrop M. Mapping complex disease traits with global gene expression. Nat Rev Genet. 2009;10(3):184-94.

34. Dimas AS, Deutsch S, Stranger BE, Montgomery SB, Borel C, Attar-Cohen H, et al. Common regulatory variation impacts gene expression in a cell type-dependent manner. Science. 2009;325(5945):1246-50.

35. Schadt EE, Molony C, Chudin E, Hao K, Yang X, Lum PY, et al. Mapping the genetic architecture of gene expression in human liver. PLoS Biol. 2008;6(5):e107.

36. Spielman RS, Bastone LA, Burdick JT, Morley M, Ewens WJ, Cheung VG. Common genetic variants account for differences in gene expression among ethnic groups. Nat Genet. 2007;39(2):226-31.

37. Stranger BE, Forrest MS, Clark AG, Minichiello MJ, Deutsch S, Lyle R, et al. Genome-wide associations of gene expression variation in humans. PLOS Genet. 2005;1(6):e78

38. Stranger BE, Forrest MS, Dunning M, Ingle CE, Beazley C, Thorne N, et al. Relative impact of nucleotide and copy number variation on gene expression phenotypes. Science. 2007;315(5813):848-53.
39. Veyrieras JB, Kudaravalli S, Kim SY, Dermitzakis ET, Gilad Y, Stephens M, et al. High-resolution mapping of expression-QTLs yields insight into human gene regulation. PLoS Genet. 2008;4(10):e1000214.

40. Stranger BE, Montgomery SB, Dimas AS, Parts L, Stegle O, Ingle CE, et al. Patterns of cis regulatory variation in diverse human populations. PLoS Genet. 2012;8(4):e1002639.

41. Stranger BE, Nica AC, Forrest MS, Dimas A, Bird CP, Beazley C, et al. Population genomics of human gene expression. Nat Genet. 2007;39(10):1217-24

42. Nica AC, Parts L, Glass D, Nisbet J, Barrett A, Sekowska M, et al. The architecture of gene regulatory variation across multiple human tissues: the MuTHER study. PLoS Genet. 2011;7(2):e1002003.

43. Fu J, Wolfs MG, Deelen P, Westra HJ, Fehrmann RS, Te Meerman GJ, et al. Unraveling the regulatory mechanisms underlying tissue-dependent genetic variation of gene expression. PLoS Genet. 2012;8(1):e1002431.

44. Nicolae DL, Gamazon E, Zhang W, Duan S, Dolan ME, Cox NJ. Trait-associated SNPs are more likely to be eQTLs: annotation to enhance discovery from GWAS. PLoS Genet. 2010;6(4):e1000888.

45. Nica AC, Montgomery SB, Dimas AS, Stranger BE, Beazley C, Barroso I, et al. Candidate causal regulatory effects by integration of expression QTLs with complex trait genetic associations. PLoS Genet. 2010;6(4):e1000895.

46. Ding J, Gudjonsson JE, Liang L, Stuart PE, Li Y, Chen W, et al. Gene expression in skin and lymphoblastoid cells: refined statistical method reveals extensive overlap in cis-eQTL signals. Am J Hum Genet. 2010;87(6):779-89.

47. Innocenti F, Cooper GM, Stanaway IB, Gamazon ER, Smith JD, Mirkov S, et al. Identification, replication, and functional fine-mapping of expression quantitative trait loci in primary human liver tissue. PLoS Genet. 2011;7(5):e1002078.

48. Gibbs JR, van der Brug MP, Hernandez DG, Traynor BJ, Nalls MA, Lai SL, et al. Abundant quantitative trait loci exist for DNA methylation and gene expression in human brain. PLoS Genet. 2010;6(5):e1000952.

49. Kabakchiev B, Silverberg MS. Expression quantitative trait loci analysis identifies associations between genotype and gene expression in human intestine. Gastroenterology. 2013;144(7):1488-96. 1496 e1481-1483.

50. Closa A, Cordero D, Sanz-Pamplona R, Sole X, Crous-Bou M, Pare-Brunet L, et al. Identification of candidate susceptibility genes for colorectal cancer through eQTL analysis. Carcinogenesis. 2014;35(9):2039-46.

51. Genomes Project C, Abecasis GR, Altshuler D, Auton A, Brooks LD, Durbin $\mathrm{RM}$, et al. A map of human genome variation from population-scale sequencing. Nature. 2010;467(7319):1061-73.

52. Ward LD, Kellis M. Interpreting noncoding genetic variation in complex traits and human disease. Nat Biotechnol. 2012;30(11):1095-106.

53. Sollid LM, Villadangos JA. Antigen processing. Curr Opin Immunol. 2013;25(1):71-3.

54. Wu S, Trievel RC, Rice JC. Human SFMBT is a transcriptional repressor protein that selectively binds the N-terminal tail of histone H3. FEBS Lett. 2007;581(17):3289-96.

55. Zhang J, Bonasio R, Strino F, Kluger Y, Holloway JK, Modzelewski AJ, et al. SFMBT1 functions with LSD1 to regulate expression of canonical histone genes and chromatin-related factors. Genes Dev. 2013;27(7):749-66.

56. He J, Wilkens LR, Stram DO, Kolonel LN, Henderson BE, Wu AH, et al. Generalizability and epidemiologic characterization of eleven colorectal cancer GWAS hits in multiple populations. Canc Epidemiol Biomarkers Prev: Publ Am Assoc Canc Res Cosponsored Am Soc Prev Oncol. 2011;20(1):70-81.

57. Biancolella M, Fortini BK, Tring S, Plummer SJ, Mendoza-Fandino GA, Hartiala J, et al.. Identification and characterization of functional risk variants for colorectal cancer mapping to chromosome 11q23.1. Hum Mol Genet. 2013.

58. Kupfer SS, Anderson JR, Hooker S, Skol A, Kittles RA, Keku TO, et al. Genetic heterogeneity in colorectal cancer associations between African and European Americans. Gastroenterology. 2010;139(5):1677-85. 1685 e 1671-1678.

59. Wang H, Haiman CA, Burnett T, Fortini BK, Kolonel LN, Henderson BE, et al. Fine-mapping of genome-wide association study-identified risk loci for colorectal cancer in African Americans. Hum Mol Genet. 2013;22(24):5048-55.

60. Wang H, Burnett T, Kono S, Haiman CA, Iwasaki M, Wilkens LR, et al. Trans-ethnic genome-wide association study of colorectal cancer identifies a new susceptibility locus in VTI1A. Nat Commun. 2014;5:4613.

61. Gamazon ER, Badner JA, Cheng L, Zhang C, Zhang D, Cox NJ, et al. Enrichment of cis-regulatory gene expression SNPs and methylation quantitative trait loci among bipolar disorder susceptibility variants. Mol Psychiatry. 2013;18(3):340-6. 
62. Davis LK, Gamazon ER, Kistner-Griffin E, Badner JA, Liu C, Cook EH, et al. Loci nominally associated with autism from genome-wide analysis show enrichment of brain expression quantitative trait loci but not lymphoblastoid cell line expression quantitative trait loci. Mol Autism. 2012;3(1):3.

63. Wheeler HE, Gamazon ER, Wing C, Njiaju UO, Njoku C, Baldwin RM, et al. Integration of cell line and clinical trial genome-wide analyses supports polygenic architecture of Paclitaxel-induced sensory peripheral neuropathy Clin Cancer Res. 2013:19(2):491-9.

64. Consortium GT. The Genotype-Tissue Expression (GTEx) project. Nat Genet. 2013:45(6):580-5.

65. Petretto E, Bottolo L, Langley SR, Heinig M, McDermott-Roe C, Sarwar R, et al. New insights into the genetic control of gene expression using a Bayesian multi-tissue approach. PLoS Comput Biol. 2010;6(4):e1000737.

66. Wang Y, Liu CL, Storey JD, Tibshirani RJ, Herschlag D, Brown PO. Precision and functional specificity in mRNA decay. Proc Natl Acad Sci U S A. 2002;99(9):5860-5.

67. Bevilacqua A, Ceriani MC, Capaccioli S, Nicolin A. Post-transcriptional regulation of gene expression by degradation of messenger RNAs. J Cell Physiol. 2003;195(3):356-72

68. Eberle AB, Stalder L, Mathys H, Orozco RZ, Muhlemann O. Posttranscriptional gene regulation by spatial rearrangement of the 3 untranslated region. PLoS Biol. 2008;6(4):e92.

69. Gaffney DJ, Veyrieras JB, Degner JF, Pique-Regi R, Pai AA, Crawford GE, et al. Dissecting the regulatory architecture of gene expression QTLs. Genome Biol. 2012;13(1):R7.

70. Heintzman ND, Hon GC, Hawkins RD, Kheradpour P, Stark A, Harp LF, et al. Histone modifications at human enhancers reflect global cell-type-specific gene expression. Nature. 2009;459(7243):108-12.

71. Brown CD, Mangravite LM, Engelhardt BE. Integrative modeling of eQTLs and cis-regulatory elements suggests mechanisms underlying cell type specificity of eQTLs. PLoS Genet. 2013;9(8):e1003649.

72. Kudaravalli S, Veyrieras JB, Stranger BE, Dermitzakis ET, Pritchard JK. Gene expression levels are a target of recent natural selection in the human genome. Mol Biol Evol. 2009;26(3):649-58.

73. Ollberding NJ, Nomura AM, Wilkens LR, Henderson BE, Kolonel LN. Racial/ ethnic differences in colorectal cancer risk: the multiethnic cohort study. Int J Cancer. 2011;129(8):1899-906.

74. Nguyen GC, Torres EA, Regueiro M, Bromfield G, Bitton A, Stempak J, et al. Inflammatory bowel disease characteristics among African Americans, Hispanics, and non-Hispanic Whites: characterization of a large North American cohort. Am J Gastroenterol. 2006;101(5):1012-23.

75. Kurata JH, Kantor-Fish S, Frankl H, Godby P, Vadheim CM. Crohn's disease among ethnic groups in a large health maintenance organization. Gastroenterology. 1992;102(6):1940-8.

76. Alexander DD, Waterbor J, Hughes T, Funkhouser E, Grizzle W, Manne U. African-American and Caucasian disparities in colorectal cancer mortality and survival by data source: an epidemiologic review. Cancer Biomark. 2007;3(6):301-13.

77. Carlson CS, Matise TC, North KE, Haiman CA, Fesinmeyer MD, Buyske S, et al Generalization and dilution of association results from European GWAS in populations of non-European ancestry: the PAGE study. PLoS Biol. 2013;11 (9):e1001661.

78. Howie BN, Donnelly P, Marchini J. A flexible and accurate genotype imputation method for the next generation of genome-wide association studies. PLoS Genet. 2009;5(6):e1000529.

79. Marchini J, Howie B, Myers S, McVean G, Donnelly P. A new multipoint method for genome-wide association studies by imputation of genotypes. Nat Genet. 2007;39(7):906-13.

80. Du P, Kibbe WA, Lin SM. lumi: a pipeline for processing Illumina microarray Bioinformatics. 2008:24(13):1547-8.

81. Lin SM, Du P, Huber W, Kibbe WA. Model-based variance-stabilizing transformation for Illumina microarray data. Nucleic Acids Res. 2008;36(2):e11.

82. Price AL, Patterson NJ, Plenge RM, Weinblatt ME, Shadick NA, Reich D. Principal components analysis corrects for stratification in genome-wide association studies. Nat Genet. 2006;38(8):904-9.

83. Shabalin AA. Matrix eQTL: ultra fast eQTL analysis via large matrix operations. Bioinformatics. 2012;28(10):1353-8.

84. Benjamini Y, Hochberg Y. Controlling the false discovery rate: a practica and powerful approach to multiple testing. J Roy Stat Soc Ser B. 1995:57(Journal Article):289-300.
85. Stegle $O$, Parts $L$, Durbin R, Winn J. A Bayesian framework to account for complex non-genetic factors in gene expression levels greatly increases power in eQTL studies. PLoS Comput Biol. 2010;6(5):e1000770.

86. Johnson AD, Handsaker RE, Pulit SL, Nizzari MM, O'Donnell CJ, de Bakker PI. SNAP: a web-based tool for identification and annotation of proxy SNPS using HapMap. Bioinformatics. 2008;24(24):2938-9.

87. Hindorff LA, Sethupathy P, Junkins HA, Ramos EM, Mehta JP, Collins FS, et al. Potential etiologic and functional implications of genome-wide association loci for human diseases and traits. Proc Natl Acad Sci U S A. 2009;106(23):9362-7.

88. Weir BS, Hill WG. Estimating F-statistics. Annu Rev Genet. 2002;36:721-50

89. Voight BF, Kudaravalli S, Wen X, Pritchard JK. A map of recent positive selection in the human genome. PLoS Biol. 2006;4(3):e72

\section{Submit your next manuscript to BioMed Central and take full advantage of:}

- Convenient online submission

- Thorough peer review

- No space constraints or color figure charges

- Immediate publication on acceptance

- Inclusion in PubMed, CAS, Scopus and Google Scholar

- Research which is freely available for redistribution 